\title{
COMPETITIVIDAD DE LOS CLÚSTER TEXTIL PERÚ MÉXICO
}

\author{
COMPETITIVENESS TEXTILE CLUSTER PERU MEXICO
}

\author{
Ana María Gutiérrez HubY* \\ Docente Principal de la Facultad de Ciencias Contables, UNMSM \\ María Eugenia Amador Murguía** \\ Profesora de la Universidad de la Guadalajara - México \\ Elsa Violeta Rafael Díaz ${ }^{* * *}$
}

Colaboradora

[Recepción: Febrero de 2011/ Conformidad: Abril de 2011]

\section{RESUMEN}

El presente artículo es una síntesis del estudio de investigación denominado "Estudio comparativo en clúster textil Perú-México para determinar estrategias competitivas" que fuera desarrollado en el año 2010 por la autora con la colaboración de un equipo de investigadores de la Universidad de Guadalajara. Este artículo básicamente presenta los resultados en términos comparativos de la situación competitiva de los clúster textil: peruano y mexicano: "Gamarra" y "Zapotlanejo” respectivamente -representativos en el rubro textil de sus países- con la intención de compartir aspectos de la dinámica operativa existente en ambos escenarios para mantener u optimizar los niveles productivos lo cual finalmente permitió al equipo de investigación el planteamiento de estrategias competitivas para su respectiva consolidación en el mercado nacional con proyección a adentrarse en el internacional.

Palabras clave: Clúster, competitividad, estrategias competitivas.

\begin{abstract}
This article is a summary of the research study entitled "A comparative study in Peru- Mexico textile cluster in determining competitive strategies" that was developed in 2010 by the author in collaboration with a team of researchers at the University of Guadalajara. This article basically presents the results in comparative terms of the competitive situation of the textile cluster, Peru and Mexico, "Gamarra" and "Zapotlanejo" respectively representing the textile industry in their countries, with the intention of sharing operational aspects of the dynamics existing in both scenarios to maintain or optimize production levels which ultimately allowed the research team's approach their respective competitive strategies for market consolidation in the national projection and international venture.
\end{abstract}

Key words: Textile Cluster, competitiveness competitive strategies.

\footnotetext{
* Magíster en Gestión Empresarial, UNMSM; Licenciada en Ciencias Administrativas. Docente investigadora de la Facultad de Ciencias Contables, UNMSM.

E-mail: anamaria_01053@yahoo.es

** Magister en Contabilidad, Docente de la Universidad de Guadalajara - México. Docente Investigadora - Universidad de Guadalajara.

E-mail: maruamador@hotmail.com *** ..
} 


\section{INTRODUCCIÓN}

La introducción del término clúster ampliamente conocido a partir del trabajo desarrollado por el Dr. Michael Porter, economista estadounidense, docente de la Universidad de Harvard, especialista en gestión y administración ha dado lugar a un sinnúmero de investigaciones orientadas al estudio de los sectores competitivos de una determinada nación.

El presente artículo aborda también esta temática, en el contexto de lo que comprende el clúster textil en dos países hermanos: Perú y México cada cual tomando la representatividad de sus conglomerados Gamarra y Zapotlanejo, respectivamente. Este tema ya abordado en la investigación denominada "Estudio comparativo en clúster textil Perú-México para determinar estrategias competitivas", se presenta de una manera sintetizada en este artículo que tiene por objetivo reseñar sus aspectos más importantes.

Siendo en la actualidad la industria textil/ confecciones una de las ramas más importantes en ambas naciones en términos de crecimiento económico y generación de empleo, el enfoque que el trabajo de investigación desarrollado presenta sobre los referidos clúster representativos del sector se orienta al propósito de proveer una base analítica y descriptiva para una definición de estrategias tendientes a visualizar el esfuerzo, el desarrollo de la productividad y la competitividad de cada uno de los clúster materia de estudio.

No obstante lo referido anteriormente cabe reseñar que este sector desde tiempo atrás ha atravesado en distintas oportunidades etapas de crisis que afectaron su auge, una de las más notables fue la crisis del modelo económico presentado a fines de los años setenta y principios de los ochenta en México y en Perú por los años 90 cuando se pasó de una economía cerrada a una abierta, situación que representó un gran reto para los productores nacionales que producían bajo medidas proteccionistas con elevados subsidios por parte del estado lo que se traducía en elevados costos de producción, obsolescencia de maquinaria y baja productividad lo que trajo para México pérdida de mercados internacionales y para el Perú bajo interés de inserción en los mercados internacionales.

Así mismo el cambio brusco de la variación de modelo económico, la liberación del mercado y posteriormente la entrada de productos asiáticos sí que representó para los productores nacionales en ambos países una competencia desleal por los bajos precios de los productos asiáticos haciendo vislumbrar difícil la situación por la creciente competencia extranjera, disminución de los márgenes de ganancias de los empresarios textiles y la obsolescencia en sus plantas productivas.

A esto se suma la crisis financiera internacional (2008) que principalmente acometió sobre los pequeños empresarios, develando en ambos países las fuertes debilidades del sector, como: la poca capacidad de asociatividad e integración para producir, falta de capital para su desarrollo, limitado uso de tecnología, baja capacitación y calificación de cuadros gerenciales y operativos, entre otros.

Pese a lo reseñado anteriormente los clúster Gamarra y Zapotlanejo en el transcurso del tiempo logran gran posicionamiento en el inconsciente colectivo de sus respectivos países como imagen de aglomeración espontánea de pequeños y medianos productores que, tras superar los inconvenientes de su inicio casi artesanal, hoy constituyen una poderosa herramienta económica que ha dinamizado el área geográfica en el que se han desarrollado pero que todavía plantean el requerimiento de la formulación de políticas e instrumentos que redunden en la optimización del sector textil y confecciones para el pequeño empresariado. 


\section{DISEÑO METODOLÓGICO}

La investigación se inscribe en el tipo de investigación básica al contribuir a incrementar el conocimiento sobre la dinámica de operación del clúster Gamarra en Perú y clúster Zapotlanejo en México, así mismo tiene carácter aplicativo o tecnológico por cuanto las estrategias de crecimiento y conclusiones reportadas sugieren mejoramiento de los clúster estudiados.

Instrumentos de recolección de datos:

- Entrevistas

- Observación

Metodología:

- Elaboración de instrumento de levantamiento de información: cuestionario.

- Aplicación del cuestionario al empresariado que opera en los respectivos clúster.

- Realización del análisis FODA con sus instrumentos MEFI Y MEFE

- Identificación y valoración de los factores críticos de éxito para determinar el perfil competitivo de los cluster.

- Formulación de estrategias

\section{GENERALIDADES DE LOS CLÚSTER GAMARRA Y ZAPOTLANEJO}

Conociendo la geografía de Perú y México, obviamente que hay disparidad en el tamaño territorial de los lugares que acogen a los clúster en estudio, por ello el estudio se ha centrado en una comparación en términos de representatividad de potencialidad comercial en el rubro de las confecciones textiles, resultando importante situar al lector en ambos escenarios.

El clúster peruano "Gamarra" ubicado geográficamente en el distrito limeño La Victoria que a su vez es uno de los 43 distritos de la provincia de Lima se sitúa en las cercanías de la ciudad capital ocupando un área de $9 \mathrm{Km}^{2}$. El clúster mexicano Zapotlanejo se desarrolla en el Municipio Zapotlanejo perteneciente geográficamente al Estado de Jalisco, se sitúa a 30 kilómetros al oriente de la capital del Estado, constituyéndose en un área de $643.02 \mathrm{~km}^{2}$.

"Gamarra" se desarrolla específicamente en un área que concentra actualmente cuarenta cuadras del distrito de La Victoria ocupando aproximadamente $0.6 \mathrm{~km}^{2}$ de los $9 \mathrm{~km}^{2}$. que tiene este distrito. Zapotlanejo que más bien se desarrolla disperso en todo el Municipio, concentra un núcleo constituido por 35 manzanas que concentran aproximadamente $0.33 \mathrm{~km}^{2}$ en el que se ubican todos los negocios relacionados con la fabricación y comercialización de prendas de vestir.

\section{Formación del Cluster Gamarra}

Narrar los inicios de la formación del emporio comercial textil más grande del Perú: Gamarra implica referirse a los inicios del distrito de La Victoria que es el lugar donde se forjó. Su creación oficial acontece el 02 de febrero de 1920 por decreto supremo del Presidente Augusto B. Leguia durante los primeros meses de su gestión.

El cierre o reestructuración de empresas textiles en La Victoria y todo Lima por los años 70, la apertura parcial de la economía a las importaciones de los años 1980-1985 (primer gobierno del Presidente Alan García Pérez ), la implementación de estrategias de reducción de personal, además de la descentralización de operaciones a través del trabajo a domicilio y subcontratación, provocaron que empresarios despidieran a sus trabajadores indemnizándolos con maquinarias de confección. De esta manera los trabajadores despedidos empezaron una empresa con- 
tando con la maquinaria y la experiencia. La subcontratación favoreció sus inicios pues consiguieron rápidamente clientes, no siendo necesario que tuvieran un local específico pues usaban sus propios domicilios; ello sumado a la venida de migrantes internos y extranjeros: italianos, árabes y chinos, que venían con la tradición de la confección, provocó el surgimiento y crecimiento del clúster Gamarra.

\section{Formación del Cluster Zapotlanejo:}

No se conoce el decreto que erigió a Zapotlanejo como municipio, pero en el Plan de División Provisional del Territorio del Estado de Jalisco, de fecha 27 de marzo de 1824, se menciona a Zapotlanejo como ayuntamiento. En noviembre del mismo año, se declara al pueblo de Zapotlanejo como cabecera del departamento de Tonalá. El decreto número 366, del 19 de septiembre de 1873, ya hace mención de Zapotlanejo como municipalidad.

En 1824 Zapotlanejo es capital del departamento de Tonalá. Desde 1825 perteneció al $1^{\circ}$ cantón de Guadalajara. Posteriormente, en 1887 Zapotlanejo forma el $4^{\circ}$ departamento del $1^{\circ}$ cantón. A Zapotlanejo, se le otorgó ayuntamiento por decreto el 8 de abril de 1844 .

Su desarrollo en el sector textil se ha fundamentado a partir de iniciativas locales que con el tiempo fueron fortaleciéndose a través de la conformación de redes sociales (familiares y amicales). En los últimos años ha transformado su base económica del sector primario al sector manufacturero; acelerado desarrollo económico que ha traído consigo transformaciones en su estructura urbana.

\section{RESULTADOS}

\section{SITUACIÓN DEL SECTOR TEXTIL/ CONFECCIONES EN PERÚ Y EN MÉXICO \\ Impacto del sector textil/confecciones en el ámbito económico del país}

Para el caso peruano el sector textil/confecciones es uno de los más importantes de la actividad puramente manufacturera y ello se corrobora con la contribución de este sector al PBI nacional manufacturero que en el año 2007 era de $7 \%$, en el 2008 de $6 \%$ y en el 2009 de $7.1 \%$.

Cabe remarcar que en este último período las exportaciones peruanas de textiles y confecciones se dirigían a los Estados Unidos en un $41 \%$, a Venezuela en un $24 \%$ y a otros mercados restantes en un $36 \%$, es entre enero y octubre del 2009 que se advierte la caída de las exportaciones textiles y confecciones a Estados Unidos en 28\% y a Venezuela en $44 \%$ respectivamente tras registrar durante cinco años consecutivos resultados positivos a una tasa promedio anual de $20 \%{ }^{1}$. A la agudización de esta crisis contribuiría la fuerte competencia de los productos asiáticos, abastecedores del $70 \%$ de la demanda estadounidense de textiles y confecciones.

Así mismo la mayoría de empresas textiles y confecciones remataron sus stocks de producción con descuentos de hasta $15 \%$ a fin de mantener el dinamismo de las compras en el mercado nacional debido a que la crisis económica afectó sus colocaciones en el exterior. A esto se sumaría la reducción significativa de la capacidad instalada para las fábricas de tejidos de punto $(-55 \%)$ y las de hilados y tejidos $(-70 \%)$, sin dejar de men-

2 Boletín No 844. Sociedad Nacional de Industria. Marzo, 2010. 
cionar que los precios se redujeron hasta en $10 \%$ como solicitud de los compradores del exterior, debido a la caída de la demanda.

En el 2010 se advirtió un crecimiento de $15.2 \%$ en el primer semestre (enero a junio) comparado con el mismo período del 2009. Aunque este aumento no fue suficiente para recuperar la fuerte caída experimentada se advirtió que el sector superaría de a pocos los efectos de la crisis externa proyectándose que a fines de año se empezarían a retomar los indicadores del 2008. La recuperación efectiva se prevé se producirá a fines del segundo trimestre del año 2011.

En lo que respecta a las exportaciones de textiles y confecciones el primer semestre del 2010 hubo una disminución de $7.34 \%$ comparado con el mismo semestre del año 2009, pasando de 718.5 millones de dólares a 665.8 millones. En este mismo período la exportación a los Estados Unidos principal mercado textil, pasó de 295.2 millones de dólares a 320.3 millones, creciendo $8.48 \%$.

En el 2010 las exportaciones estuvieron dirigidas a nuevos mercados como Colombia y Ecuador, no obstante la pérdida de las exportaciones al mercado venezolano significará que se exporte US\$ 130 millones menos que el 2009.

Internamente se advierte que las confecciones peruanas están ganando terreno a las importaciones en las tiendas por departamento debido a su mejor calidad y diseño; en este sentido, existen muchas empresas peruanas que le están vendiendo a tiendas comerciales como Ripley; la Sociedad Nacional de Industrias está tratando de certificar a más de estas empresas que están en la capacidad de fabricar más prendas para este minorista que maneja alrededor de ochenta marcas peruanas en la actualidad. Esta inclu- sión se debe en gran parte a la demanda de los consumidores que reconocen el talento y la creatividad de los diseñadores nacionales.

En el caso Mexicano la industria textil destaca su participación entre las otras industrias manufactureras en un 5\% del Producto Interno Bruto Manufacturero, dato correspondiente al segundo trimestre del año 2009, la disgregación de esta cadena productiva en los rubros textil-vestido-cuero expresa como datos porcentuales: textil (1.3\%), vestido $(2.3 \%)$ y cuero $(1.2 \%)$, por lo que se asevera entonces que la producción de ropa en México constituye el principal soporte de esta cadena productiva. ${ }^{3}$

El número de empresas pertenecientes al rubro textil que registró México en mayo 2009 fue de 2,136 y en junio 2,139, y dedicadas exclusivamente al rubro confección o vestido 9,602, según datos del Instituto Nacional de Estadística y Geografía - INEGI.

\section{Impacto del sector textil/confecciones en el ámbito laboral a nivel país}

En el caso peruano las confecciones peruanas en general registran una población económicamente activa (PEA) de 473,000 personas, porcentualmente: $36.5 \%$ de la PEA industrial del país e indirectamente generan un millón de empleos, según las estadísticas 2009 del Comité de la Sociedad Nacional de Industrias del país.

La rama textil de "fabricación de prendas de vestir, excepto prendas de piel" concentra la más alta proporción de trabajadores, 33 mil 488 personas, es decir $47,3 \%$ del total que concentra el sector textil en general.

En el caso Mexicano la industria de la confección contribuye activamente en la generación de empleo. La cifra de empleabili-

3 Fuente: Informe de la Cámara Nacional de la Industria del Vestido e INEGI-México. 
dad que aporta esta industria alcanza un total de 391,952 empleos que constituyen el 11\% frente al resto de las otras industrias manufactureras (89\%) según datos que hasta junio del 2009 reportaba el Instituto Mexicano de Servicio Social (IMSS) difundidos por la Cámara Nacional de la Industria del Vestido de México. Este 11\% disgregado en los rubros textil y vestido alcanza $3 \%$ para el primer rubro con 104,102 empleos y $8 \%$ para el rubro vestido que alcanza 287,940 puestos de trabajo, los que hacen un total de 391,952 para el sector textil/vestido.

En cuanto a la cantidad de empresas del rubro textil y vestido en general se reportó hasta junio del 2009 un registro de 11,670 empresas, comprendiendo el sector vestido 9531 empresas y el sector textil 2,139.

Otro aspecto adicional con respecto a la empleabilidad que genera el sector es la contribución activa de los diferentes Estados del país en su desarrollo, es así que el Distrito Federal, Estado de México y Puebla son los que reportan el mayor número de empleos del sector, así mismo Jalisco el Estado que alberga al clúster Zapotlanejo objeto del presente estudio, generó el $4.5 \%$ del total de empleos registrados para el sector hasta junio del año $2009(287,940)$.

\section{IMPACTO DEL CLÚSTER GA- MARRA YZAPOTLANEJO EN SUS RESPECTIVOS PAÍSES.}

\section{En el aspecto económico}

En el aspecto económico el clúster Gamarra es considerado actualmente el principal emporio del sector confecciones en el Perú por movilizar más de 800 millones de dólares al año, concentrando en esta cifra cerca del $60 \%$ de todo el sector textil y confecciones del país destinado al mercado in- terno que según cifras del Comité Nacional de Industrias (SIN) fue de 1,400 millones en el período 2009.

$\mathrm{Al}$ recibir diariamente un promedio de 250 mil visitantes, registra el mayor número de transacciones comerciales por minuto en el país, estimándose que el $60 \%$ de sus clientes son consumidores finales, el $27 \%$ empresas comercializadoras, el $11 \%$ empresas manufactureras, el $1.71 \%$ empresas de servicios, el $0.95 \%$ empresas subcontratantes y el $0.57 \%$ empresas e instituciones del estado.

El clúster mexicano, Zapotlanejo, es también una importante fuerza comercial que actualmente conforma la ruta de la moda del Estado de Jalisco que produce el $8 \%$ de las prendas de vestir a nivel nacional para abastecer al mercado interno mexicano del sector.

\section{En el aspecto laboral}

En el clúster Gamarra operan unos diez mil empresarios que dan empleo a unas 60 mil personas. Los resultados de la encuesta aplicada por el Ministerio de trabajo a 391 MYPES del clúster Gamarra reflejaron que el clúster tiene perfil de microempresa pues el 95.7\% de las empresas encuestadas refirieron tener de uno a diez trabajadores/as, el $82 \%$ de uno a cuatro trabajadores y el $30 \%$ dos trabajadores.

Se observó también en esta encuesta que las MYPE del clúster Gamarra concentran un $37 \%$ de empresarios y trabajadores nacidos en Lima y $62 \%$ nacidos en 22 departamentos del país, principalmente Junín, Puno, Ancash y Cajamarca.

En el caso del clúster Zapotlanejo el dinamismo de su producción y comercio brinda empleo a 3,500 personas. La actividad comercial de este clúster representa el $41 \%$, la actividad manufacturera el $14 \%$ y ambas actividades el $45 \%$. 


\section{En el aspecto social}

El clúster Gamarra en sus inicios albergó a mucha población (migrantes internos y extranjeros) que de una u otra manera se dedicaban a la misma actividad: la textil. En la actualidad este clúster se encuentra posicionado en el inconsciente colectivo de la sociedad peruana, en especial de la limeña. En sus inicios este centro textil estaba generalizado para los sectores populares, más actualmente este mismo centro textil le surte mercaderías a grandes tiendas como Saga Falabella y Ripley, motivo por el cual el clúster Gamarra ha cambiado su estatus, recibiendo en sus locales no sólo a los "pobres" sino también a los "no tan pobres" y hasta a la gente adinerada que encuentra en las confecciones de Gamarra la misma calidad y más bajo precio que en las tiendas antes mencionadas.

Por otra parte, el clúster Zapotlanejo de México se ha consolidado como un centro productor y comercializador de prendas de vestir ganándose denominaciones como "Zapotlanejo la cuna del vestir" o "Zapotlanejo vistiendo a México".

\section{DINÁMICA DE OPERACIÓN DE LOS CLÚSTER GAMARRA Y ZAPOTLANEJO}

La información proporcionada por los empresarios del clúster Gamarra y Zapotlanejo, facilitó la obtención del diagnóstico situacional y la identificación de fortalezas, debilidades, oportunidades y amenazas que permitieron la selección final de 16 factores internos y externos, determinantes para el éxito de las respectivas pymes y la demostración de su competitividad en el mercado.

\section{Infraestructura exportadora}

La infraestructura exportadora es el primer factor crítico de éxito identificado. A este respecto cabe señalar que las PYMES del clúster Gamarra no cuentan con la suficiente implementación de activos para una producción masiva, lo que constituye una gran debilidad para el clúster. En cuanto el clúster Zapotlanejo también presenta la misma debilidad pues la mayoría de las empresas constituidas son PYMES que no cuentan con la tecnología de punta, ni con los recursos suficientes para producir a gran escala y ello no les permite exportar.

\section{Apoyo del gobierno}

El factor "apoyo del gobierno" a favor de las PYMES considerado también clave para el éxito de las PYMES fue catalogado como una debilidad mayor común en ambos clúster.

Para el caso de Perú, las últimas legislaciones emitidas para las pequeñas empresas han demostrado que no han sido de gran ayuda para su consolidación, crecimiento o inicio de actividades, los dispositivos emitidos en la ley No 28015 (0307) ponen de manifiesto las disconformidades del sector, por lo que se considera que es otra de las debilidades mayores que presenta el clúster.

En el caso de México, a pesar que el actual gobierno a nivel nacional y a nivel del Estado de Jalisco hicieron pronunciamientos a favor de fortalecer a las PYMES y promover la incubación de empresas a través de jóvenes emprendedores de las instituciones de Educación Superior; los fondos institucionales creados para este fin, son manejados a criterio de funcionarios que deciden favorecer muchas veces a quienes menos lo necesitan.

\section{Administración capacitada}

La administración capacitada se presenta como fortaleza en ambos clúster. 
Para el caso del clúster Gamarra se categoriza como una fortaleza menor ya que a pesar de que en su gran mayoría los comercios de Gamarra no cuentan con profesionales especialistas en las diferentes actividades de la empresa, se ha podido observar también que existe preocupación por parte de diversos sectores del estado por consolidar la preparación de los empresarios para fortalecer el sector de la pequeña empresa.

En el caso de las empresas del clúster Zapotlanejo estas se han mantenido por muchos años y han pasado de generación en generación por lo que el empresario ha aprendido de sus antecesores, lo que les ha permitido tener los conocimientos suficientes y la capacidad para seguir manejando el legado de sus familias.

\section{Transparencia y valores}

El aspecto de la transparencia y los valores en la realidad de ambos clúster se presenta como una debilidad menor para el clúster Gamarra ya que las continuas batidas por la Superintendencia Nacional de Administración Tributaria (SUNAT) a la que se ve sujeta el clúster de Gamarra evidencian que permanentemente se falsifican y adulteran sus productos los cuales los clientes usuarios consideran que son legítimos. Se ha podido comprobar porque existen fábricas que elaboran etiquetas las cuales son aplicadas a productos de fabricantes desconocidos que las utilizan como si fueran productos de marcas registradas.

En el clúster mexicano el empresario de Zapotlanejo más bien refiere compromiso con su actividad y el negocio que le ha sido heredado por lo que se le observa una fortaleza menor.

\section{Ubicación de áreas funcionales}

En el caso del clúster Gamarra, este aspecto es considerado una debilidad menor puesto que tienen desconcentradas sus principales áreas funcionales como alta dirección y producción

Para el caso del clúster Zapotlanejo también es considerada una debilidad menor, ya que no tienen determinadas sus áreas específicas observándose que la mayor parte de los empresarios utilizan sus casas o pequeños talleres para fabricar sus productos y así evitar costos adicionales como renta y pago de servicios, además de pagar los impuestos correspondientes, lo cual encarece de sobremanera el producto.

\section{Oferta y promoción de productos inno- vados continuamente}

La oferta y promoción de productos innovados continuamente se presenta como una fortaleza mayor en ambos clúster. En el caso del clúster Gamarra el rubro principal es la venta y para mejorar su participación en el mercado continuamente ejecuta innovaciones en sus productos basándose en modas internacionales estacionales lo que le permite presentar productos de actualidad.

En el caso del clúster Zapotlanejo, los empresarios constantemente están innovando, acuden a ferias nacionales e internaciones por lo que sus modelos se actualizan continuamente. Son innovadores, los aparadores y maniquíes muestran la ropa de moda que gusta a los jóvenes y a los no tan jóvenes. Están trabajando conjuntamente con universidades de la ciudad de Guadalajara para que los alumnos de diseño de modas participen en eventos conjuntos, esto con la finalidad de promover sus productos de moda a precios que se consideran inigualables. 


\section{Diversidad de productos}

En cuanto a la diversidad de productos ambos clúster presentan fortaleza. Gamarra siempre ha servido a los mercados sobre la base de actualización y multidiversidad de productos para todas las líneas y edades lo cual se convierte en una fortaleza que le permite ser buscada como centro que ofrece lo último en la moda y precios.

En Zapotlanejo existe una gran diversidad de productos que se ofrecen en la zona del vestir como línea de damas, caballeros, niños, lencería, blancos, accesorios y calzado; esto se convierte en una fortaleza para los empresarios porque el cliente puede encontrar ropa para toda la familia ya que es un mercado en donde acuden familias completas en su gran mayoría. Las familias que acuden en gran medida pueden satisfacer sus demandas a precios que los hacen volver a visitar la Cuna del Vestir.

\section{know-how y experiencia manufacturera para la exportación}

En el clúster Gamarra el Know-how y la experiencia manufacturera para la exportación existe, lo que le enfrenta al problema de una exportación mayor es la poca capacidad de asociatividad que tienen los pequeños empresarios de fabricar grandes volúmenes para colocarlos en los mercados internacionales.

Ello no viene sucediendo con el empresariado del clúster Zapotlanejo los cuales si bien tienen gran experiencia en su ramo debido a que conocen el giro desde que lo tenían sus bisabuelos, abuelos y sus padres, así como know-how, el problema es que la visión del empresario hasta ahora es colocar sus productos en el mercado nacional, algunos de ellos maquilando a grandes marcas como Copel y Cklass. Por otro lado no tienen la capacidad instalada ni la capacidad financiera para proyectarse hacia la exportación.

\section{Producción por estaciones}

En este aspecto ambos clúster presentan una fortaleza mayor.

Gamarra se ha distinguido por ser el centro donde se ofertan permanentemente productos de moda y estacionales, el producto diseñado para la estación cubre las expectativas para el mercado en lo referente a la moda y a los costos de fácil acceso a todos los niveles socioeconómicos y por lo tanto es una fortaleza mayor.

Zapotlanejo siempre está a la vanguardia de la moda. Cada vez se pueden apreciar prendas más sofisticadas con adornos de piedras, serigrafía, bordados, etc'. Con oportunidad cambian los aparadores a la moda de cada estación. Los fines de semana es un tránsito incontable de personas que visitan toda la zona del vestir; y la temporada que se acerca suele ser muy visitada por tapatíos, jaliscienses y mexicanos de toda la república.

\section{Personal capacitado y entrenado}

El factor "personal capacitado y entrenado" constituye una fortaleza mayor en la realidad de ambos clúster.

En el clúster Gamarra el personal que ejecuta la línea de producción ha sido entrenado a través de los años y desarrolla sus habilidades técnicas con alta competitividad lo que le permite mantener niveles de producción y de ventas. Así mismo, el sector estatal viene desarrollando políticas de impulso en cuanto a la capacitación y adiestramiento de personal para las pequeñas y medianas empresas, alguna de las cuales se encuentran ubicadas en el mismo clúster con lo cual se impulsa la capacidad de sus recursos humanos. 
En el clúster Zapotlanejo el personal está capacitado para desarrollar sus funciones de producción, ya que tienen varios años en el giro, además de que el gobierno municipal y estatal así como organismos independientes ofrecen cursos de capacitación sobre todo en áreas de diseño, imagen corporativa y motivación de personal; esto ha sido bien visto por parte de los empresarios que han respondido con entusiasmo a este tipo de actividades.

\section{Calidad de producto}

La calidad del producto de Gamarra se considera como fortaleza mayor porque cuenta entre otros con materia prima nacional de primera calidad, reconocida en el mundo como es el algodón Pyma, el cual le da la calidad al producto en su línea de textiles de algodón.

Como contraparte de ello, la importación de materia prima textil china le resta calidad a algunos de sus productos, pero a pesar de ello sigue teniendo una alta demanda por su precio de venta.

Zapotlanejo ofrece productos de buena calidad, lo que se considera una fortaleza para el empresariado en general. La mayor parte de la materia prima que consumen es adquirida en el mercado nacional; aunque para la fabricación de ciertas prendas existe la necesidad de importar tela de la ciudad de Los Ángeles California para que las prendas tengan la calidad requerida. La materia prima importada es de una empresa cuyos propietarios son empresarios judíos.

\section{Capacidad tecnológica}

En cuanto a la capacidad tecnológica hay disparidad en la realidad de ambos clúster.

En el caso de los fabricantes del clúster Gamarra generalmente no cuentan con tec- nología de punta, siendo esta la principal limitación para generar los grandes volúmenes de producción que requiere el mercado internacional, constituyendo ello una debilidad mayor del clúster.

En el caso de los empresarios fabricantes del clúster Zapotlanejo, los mismos no cuentan con tecnología de punta, pero sí han invertido en maquinaria nueva lo que les permite producir una mayor cantidad de prendas a un menor costo y en menor tiempo aunque con proyección al mercado nacional, considerándose que ello representa una fortaleza menor para el clúster.

\section{Competitividad de precios}

Existe coincidencia en cuanto a la competitividad de los precios ofrecidos por ambos clúster. En el caso del clúster Gamarra estos son reconocidos a nivel nacional como altamente competitivos porque presentan productos de fácil alcance a todos los estratos socioeconómicos del país.

Para el caso del clúster Zapotlanejo los precios ofertados son atractivos y competitivos con otras zonas del país de giro similar, muestra de ello es la afluencia de personas.

\section{Capacidad de respuesta a la demanda nacional}

En cuanto a la capacidad de dar respuesta a la demanda nacional ambos clúster presentan esta fortaleza ya que abastecen a los clientes locales y del interior del país.

\section{Capacidad de respuesta a la demanda internacional}

El clúster Gamarra no cuenta con suficiente capacidad de respuesta ante demanda internacional debido a su limitada capacidad 
productiva, presentando en este aspecto una marcada debilidad.

Por su parte el clúster Zapotlanejo también presenta esta debilidad pues no se ha visualizado en un entorno internacional básicamente porque son microempresarios en su mayoría y no tienen la capacidad de producción ni la capacidad financiera para crecer y pensar en exportar a otros países.

\section{Medios de comunicación a los clientes}

En cuanto a la comunicación con los clientes ambos clúster presentan fortaleza en este aspecto pues en la actualidad vienen siendo utilizadas nuevas formas de comunicación como las páginas web y blogs de los diferentes consorcios establecidos en los clúster que le facilitan la aproximación a los clientes y proveedores. Así mismo la promoción tradicional que es la recomendación de un cliente a otros clientes debido al buen trato, precio, calidad y otros factores; en el caso específico del clúster Zapotlanejo el empresario está utilizando el tele marketing mailing y agencias de viajes que promocionan la visita a Zapotlanejo y otras ciudades cercanas como la ciudad de Guadalajara, Jalisco.

\section{ESTRATEGIAS COMPETITIVAS DE LOS CLÚSTER GAMARRA Y ZAPOT- LANEJO}

Como acciones para crear una posición defendible en el mercado se determinaron las siguientes estrategias las que fueron clasificadas en a) estrategias aplicables a ambos clúster, b) aplicables sólo al clúster Gamarra y c)aplicables sólo al clúster Zapotlanejo:

\section{a) Estrategias aplicables para ambos clús- ter.}

Estrategias de desarrollo de mercado:

Ambos clúster deben aplicar una estrategia de desarrollo de mercado, en el caso del clúster Gamarra esta consistiría en:

- Buscar nuevos mercados en otras áreas geográficas para contrarrestar en algunas oportunidades los embates del fenómeno del niño.

- En el caso del Clúster Zapotlanejo, este tipo de estrategia a aplicar debe orientarse a la tarea de participar en ferias nacionales e internacionales de diseño de modas y lanzar sus modelos con oportunidad estacional.

Estrategias intensivas de desarrollo de producto:

En el caso del clúster Gamarra, se proponen cuatro acciones estratégicas:

- Innovar para potenciar los equipos y poder elevar los niveles de producción.

- Innovar el producto acondicionándolo al cambio climático presentado.

- Incrementar la demanda ante clima existente. Producción por estaciones.

- Crear o utilizar portales verticales a través de la segmentación por productos concretos o para seguir estrategia de crecimiento en el mercado.

En el caso del clúster Zapotlanejo, la línea de acción se orientaría a:

- Aprovechar la calidad del diseño de los estudiantes para que conjuntamente con la calidad de la confección se puedan ofrecer productos al mercado a precios bajos que puedan competir con la importación.

Estrategia de asociatividad:

En este sentido, para el clúster Gamarra se propone: 
- Asociatividad de pequeños empresarios productores para generar grandes volúmenes de producción estandarizadas. Para el clúster Zapotlanejo se presentan dos acciones estratégicas en el marco de la asociatividad:

- Realizar compras en grupo o por asociación para que por volumen se obtengan mejores precios los que puede beneficiar al consumidor final, ofreciendo un mejor precio con un producto de calidad.

- Al integrarse como una asociación les permitirá una inversión mayor en tecnología de punta y así pensar en cubrir además del mercado nacional, exportar a otros países.

Estrategia de desarrollo de recursos humanos:

En cuanto a la optimización del recurso humano, el clúster Gamarra debería seguir la estrategia de:

- Mejorar la cultura organizacional a través de capacitación y aleccionamiento de nuevos hábitos y costumbres de vida.

- Así mismo, mejorar la calificación del personal permanentemente a través de un acercamiento con los diferentes sectores del estado afines a la pequeña empresa a través de convenios firmados para su optimización.

El clúster Zapotlanejo:

- Fortalecer mediante cursos de capacitación la formación y valores que se inculcan en el seno familiar para fomentar el trabajo en equipo, motivación, atención al cliente y demás herramientas que ayuden al crecimiento de la demanda.

Estrategias expansivas:

Para este tipo de estrategia también aplicable a ambos clúster, se plantean cuatro ac- ciones para el clúster Gamarra y una para el clúster Zapotlanejo.

\section{Clúster Gamarra:}

- Crear y/o aperturar nuevos mercados que permitan captar nuevos conocimientos globales.

- Expandir el tamaño de planta para ponerla al servicio de tercerización a nivel nacional.

- Expandir las líneas de productos no desarrollados completamente por los competidores para cubrir nichos de mercado no explorados.

- Buscar el posicionamiento de marca en nuevos mercados.

\section{Clúster Zapotlanejo:}

- Incursionar en nuevos mercados a través de servicios de maquila a grandes empresas o venta directa a mayoristas y menudeo para aprovechar la experiencia y capacidad administrativa y de mano de obra.

\section{Estrategias defensivas:}

Gamarra:

- Hacer riesgo compartido (Joint venture) con socios estratégicos para el ingreso a nuevos mercados.

Zapotlanejo:

- Solicitar al gobierno elevar el arancel en la importación de prendas de vestir para disminuir la invasión de productos asiáticos.

- Buscar apoyo de Bancomex para que se integre una Asociación de fabricantes $y$ en el mediano plazo poder exportar a otros países. 


\section{b) Estrategias aplicables al clúster Gama-} rra:

Estrategias de integración vertical hacia atrás:

- Tercerizar servicios a empresas globales.

Estrategias de diferenciación:

- Diferenciar el producto elitizándolo para servir a un segmento específico alto.

Estrategia de integración horizontal:

- Mejorar los niveles de educación difundiendo los beneficios de la asociatividad como elemento importante de respuesta al embate externo.

Estrategia de liderazgo de costos.

- Establecer estrategia en liderazgo de costos y de especialización mediante la diferenciación de sus productos dentro de un segmento de mercado.

Estrategia de diversificación de productos:

- Establecer políticas de acuerdo a nichos específicos aprovechando la diversidad de productos, su know-how en la fabricación y experiencia manufacturera para ingresar a nuevos mercados.

Estrategia de innovación:

- Mantener constante innovación con calidad en el producto buscando la satisfacción estacional del cliente.

\section{c) Estrategias aplicables al clúster Zapot- lanejo:}

Estrategia de integración vertical hacia delante:

- Establecer convenios con empresas a través de los TLC para ofrecer servicios de maquila para que pueda generarse fuente de empleo que a su vez permitan seguir capacitando la mano de obra de la confección.

Estrategia de comunicación corporativa:

- Generar diálogo con las autoridades tanto del gobierno central como del federal para promover legislación que contemple sanciones para evitar la importación ilegal que perjudica a la industria nacional.

- Generar campañas a nivel nacional conjuntamente con la Secretaria de Salud para crear conciencia entre la población de los riesgos de adquirir ropa importada de dudosa procedencia.

- Ampliar el catálogo de productos de tal forma que quien acuda a realizar sus compras encuentre ropa para "vestirse de la cabeza a los pies". Para dar esta satisfacción al cliente es necesario que realice contratos o convenios con fabricantes de las líneas que no se producen en Zapotlanejo, para que los precios sean atractivos y el producto de calidad.

- Utilizar la web para subir información acerca de los que es "La Cuna del vestir", como una herramienta muy utilizada por los jóvenes empresarios lo cual potenciará una mejora alternativa de negocio.

Estrategia de alianza estratégica:

- Establecer convenios con instituciones de educación superior para que los estudiantes de la carrera de diseño de modas hagan propuestas de diseños innovadores estacionales al clúster.

Estrategia de diversificación de proveedores:

- Buscar proveedores alternativos que les ofrezcan una mayor variedad de telas de acuerdo a la prenda que se fabrica ya que es parte del control de calidad y por ende del precio de venta. 


\section{CONCLUSIONES}

- Con relación al Análisis Competitivo de los clúster Gamarra - Zapotlanejo, se establecieron los Factores Críticos de Éxito (FCE) más importantes: cuatro FCE relacionados a Gestión Empresarial (Infraestructura exportadora, apoyo del gobierno, administración capacitada, transparencia y valores), tres FCE en Administración (Ubicación de áreas funcionales principales como alta dirección, producción y ventas; ofertas y promoción de productos innovados continuamente; diversidad de productos), cinco FCE en Producción (Know How y experiencia manufacturera exportadora del sector, Producción por estaciones, Personal capacitado y entrenado, calidad del producto y capacidad tecnológica) y cuatro FCE en Ventas (competitividad de precios, capacidad de respuesta a la demanda interna, capacidad de respuesta al mercado internacional, medios de comunicación con los clientes).

- La ponderación de los factores críticos de éxito para el caso de "Gamarra" (3.33), refleja que "Gamarra" se encuentra fuerte aunque menos competitiva en comparación con la ponderación alcanzada por “Zapotlanejo" (3.71) que se presenta un poco más fuerte.

- Así mismo del análisis de los pesos asignados a los Factores Críticos de Éxito se observó que el Clúster Peruano "Gamarra", aventaja a "Zapotlanejo" en los factores "Administración" y "Gestión" lo que denota que en Perú existe una mayor preocupación por los aspectos administrativos que en México; por otra parte, en relación a los factores de "Producción" y "Ventas" se observa que Zapotlanejo considera mayor peso para esta área que
Gamarra, lo que demostraría que el clúster Zapotlanejo presenta una mejor distribución de las áreas que componen la empresa.

- En el aspecto del reforzamiento de la gestión de las MYPE del Clúster Gamarra hay intervención del Estado en cuanto al ámbito de la capacitación de mano de obra a través de sus diferentes sectores involucrados en la microempresa; lo que no viene ocurriendo en el caso de Zapotlanejo en el que la capacitación de mano de obra se adquiere en forma de práctica laboral.

- En ambos clúster el apoyo gubernamental no ha tenido el impacto esperado ya que presentaron similares problemáticas: a) exceso de documentos solicitados; b) dificultad para encontrar los avales a su solicitud de crédito; c) altas tasas de interés manejadas en los préstamos.

- Se presentan aspectos antagónicos relacionados con una cultura organizacional individualista, en la que al mismo tiempo procura construir y mantener redes de cooperación tanto sociales como empresariales, formales o informales.

- En cuanto a las capacidades exportadoras se concluyó que Gamarra sí desarrolla su potencial exportador $y$ tiene consolidado su mercado a nivel nacional, mientras que el clúster Zapotlanejo se maneja sólo a nivel de mercado nacional.

\section{RECOMENDACIONES}

- A la luz de los resultados se recomienda que en el clúster Zapotlanejo se impulse mejor el área de gestión y el clúster Gamarra realice lo propio con las áreas de Producción y Ventas a fin de que ambas desarrollen mejor sus estrategias competitivas. 
- Se recomienda el reforzamiento de los niveles de capacitación en gestión y operación con intervención estatal de manera que les permita optimizar tecnología, mejorar gestión, e impulsar la innovación en cuanto a sus procedimientos para manufactura y comercialización de productos.

- Implementar una ventanilla única para trámites (VUT) en general de las MYPE tanto para México como para Perú. En especial al caso de Perú lo que permitiría potenciar su actuación porque se impediría la dilación en los trámites.

- Es también conveniente procurar construir y mantener redes de cooperación tanto social como empresarial, formal e informal con la finalidad de ayudar a mejorar la cultura organizacional imperante en ambos clúster.

- Establecer redes de comunicación empresarial para optimizar la comercialización estacional para su estratificación y diversificación en beneficio de potenciar la exportación.

- Al evidenciar Zapotlanejo una fuerte desventaja en cuanto a su potencial exportador, debería incidir en una mayor capacitación para adquirir mayores conocimientos en el rubro exportación lo que le permitiría un mayor crecimiento y generación de mayor empleo.

\section{REFERENCIAS BIBLIOGRÁFICAS}

1. ASOCIACIÓN DE EXPORTADORES - ADEX. Revista de la Asociación de Exportadores del Perú. Edición No 364.

2. ASOCIACIÓN DE EXPORTADORES - ADEX. Revista de la Asociación de Exportadores del Perú. Edición No 363.

3. ASOCIACIÓN DE EXPORTADORES - ADEX. Revista de la Asociación de Exportadores del Perú. Edición No 362.
4. CÁMARA NACIONAL DE LA INDUSTRIA DEL VESTIDO. Situación actual de la industria del vestido en México. 2009.

5. CARRIÓN MAROTO, Juan. Estrategia de la Visión de la Acción. Ed. Libros Profesionales de Empresa. 2da. Edición. Madrid, 2007.

6. DALESSIO IPINZA, Fernando. El Proceso Estratégico - Un enfoque de Gerencia. Edit. Pearson. 1ra. Edición. 2008.

7. GARCÍA RODRÍGUEZ, M. Un modelo de análisis competitivo del sector farmacéutico. Investigaciones Europeas de Dirección y Economía de la Empresa. Vol. 1 No 3, 1995, pp. 103-111.

8. GARRIDO BUJ, Santiago. Dirección Estratégica. Edit. Mc Graw Hill. 2da. Edición. España. 2006.

9. INSTITUTO NACIONAL DE ESTADÍSTICA E INFORMÁTICA - INEI - PERÙ. http://www.inei.gob.pe/

10.INSTITUTO NACIONAL DE ESTADÍSTICA Y GEOGRAFÍA - INEGI MÉXICO. Indicadores Macroeconómicos de Coyuntura. Recuperado de página web: http://dgcnesyp.inegi.org.mx/cgiwin/bdiecoy.exe/492? s=est \&c $=12343$

11.MINISTERIO DE TRABAJO Y PROMOCIÓN DEL EMPLEO. Aportes para la construcción de una agenda sobre descentralización y desarrollo económico local. Perú. 2008

12.MINISTERIO DE TRABAJO Y PROMOCIÓN DEL EMPLEO. Necesidades de Servicios de Desarrollo Empresarial de las MYPE de Confecciones en Gamarra. Perú. 2008

13.MINISTERIO DE TRABAJO Y PROMOCIÓN DEL EMPLEO. Aspiraciones y perspectivas de empresarios y empresarias de Gamarra. Perú. 2008 
Ana María Gutiérrez Huby, Elsa Violeta Rafael Diaz

14.SOCIEDAD NACIONAL DE INDUSTRIAS. Boletín No 840-841. Edición Especial.2009.

15.SOCIEDAD NACIONAL DE INDUSTRIAS. Boletín Edición No 849-850/ Setiembre-octubre 2010.
16.SOCIEDAD NACIONAL DE INDUSTRIAS. Boletín Edición No 848 / Agosto 2010.

17.SOCIEDAD NACIONAL DE INDUSTRIAS. Boletín Edición No 844 / Marzo 2010. 